Article

\title{
Mobility as a Service: A Critical Review of Definitions, Assessments of Schemes, and Key Challenges
}

\author{
Peraphan Jittrapirom ${ }^{1, *}$, Valeria Caiati ${ }^{2}$, Anna-Maria Feneri ${ }^{2}$, Shima Ebrahimigharehbaghi ${ }^{1}$, María J. Alonso- \\ González ${ }^{3}$ and Jishnu Narayan ${ }^{3}$ \\ ${ }^{1}$ Nijmegen School of Management, Radboud University, 6500 HK Nijmegen, The Netherlands; \\ E-Mails: p.jittrapirom@fm.ru.nl (P.J.), s.ebrahimi@fm.ru.nl (S.E.) \\ 2 Department of the Built Environment, Urban Planning Group, Technische Universiteit Eindhoven, 5600MB Eindhoven, \\ The Netherlands; E-Mails: V.Caiati@tue.nl (V.C.), A.Feneri@tue.nl (A-M.F.) \\ ${ }^{3}$ Faculty of Civil Engineering and Geosciences, Technische Universiteit Delft, 2628 CN Delft, The Netherlands; E-Mails: \\ M.J.AlonsoGonzalez@tudelft.nl (M.J.A-G.), J.N.SreekantanNair@tudelft.nl (J.N.)
}

* Corresponding author

Submitted: 28 February 2017 | Accepted: 2 June 2017 | Published: 29 June 2017

\begin{abstract}
Mobility as a Service (MaaS) is a recent innovative transport concept, anticipated to induce significant changes in the current transport practices. However, there is ambiguity surrounding the concept; it is uncertain what are the core characteristics of MaaS and in which way they can be addressed. Further, there is a lack of an assessment framework to classify their unique characteristics in a systematic manner, even though several MaaS schemes have been implemented around the world. In this study, we define this set of attributes through a literature review, which is then used to describe selected MaaS schemes and existing applications. We also examine the potential implications of the identified core characteristics of the service on the following three areas of transport practices: travel demand modelling, supply-side analysis and business model design. Finally, we propose the necessary enhancements needed to deliver such an innovative service like Maas, by establishing the state of art in those fields.
\end{abstract}

\section{Keywords}

business model; innovative mobility services; integrated mobility; modelling

Issue

This article is part of the issue "Smart Cities - Infrastructure and Information", edited by Soora Rasouli, Harry Timmermans and Dujuan Yang (Eindhoven University of Technology, The Netherlands).

(C) 2017 by the authors; licensee Cogitatio (Lisbon, Portugal). This article is licensed under a Creative Commons Attribution 4.0 International License (CC BY).

\section{Introduction}

In recent years, the increasing number of transport services offered in cities and the advancements in technology and ICT have introduced an innovative Mobility as a Service (MaaS) concept. It combines different transport modes to offer a tailored mobility package, similar to a monthly mobile phone contract and includes other complementary services, such as trip planning, reservation, and payments, through a single interface (Hietanen, 2014). This bundling of mobility modes presents a shift away from the existing ownership-based transport sys- tem toward an access-based one. It offers users a tailored hyper-convenient mobility solution, with a promising perspective to substitute private car.

Given its promising prospects, there is still a high degree of ambiguity surrounding the concept with multiple sources vying to offer definitions of Maas, many of which may conflict with one another or deal with different aspects of the concept altogether. Additionally, although several MaaS schemes have been implemented around the world, there is a lack of assessment framework that classifies their unique characteristics in a systematic manner. "What constitutes a MaaS concept?" 
is the central proposition of this study. To examine this proposition, we first attempt to define Maas and the core characteristics based on a literature review. We build on earlier related works, such as Kamargianni, Li, Matyas and Schäfer (2016) and report our overview in Section 2. We then use these characteristics to review 12 selected MaaS schemes, presented in Section 3. This reveals certain differences and similarity trends among the schemes considered. It also grounds our theoretical characteristics of MaaS at an operational level and reveals certain attributes unique to a practical level. Next, we highlight the challenges to approach this emerging phenomenon of MaaS in Section 4, by examining the potential implications of the identified core characteristics of the service on the following three areas of transport practices: travel demand modelling, supply-side analysis, and designing business model. We review the state of the art in the three areas and explore the probable enhancements that will be required to deliver such an innovative and integrated mobility service. Finally, the paper is concluded in Section 5. The outcomes can be useful to pinpoint MaaS' core characteristics, derive a framework to assess MaaS schemes that fulfil to a certain degree this set of attributes and to indicate future challenges in transportation research.

\section{Definition of MaaS}

\subsection{Existing Definitions}

Mobility as a Service (MaaS) is a very recent mobility concept. It can be thought of as a concept (a new idea for conceiving mobility), a phenomenon (occurring with the emergence of new behaviours and technologies) or as a new transport solution (which merges the different available transport modes and mobility services). The first comprehensive definition of MaaS is offered by $\mathrm{Hi}$ etanen (2014). He describes MaaS as a mobility distribution model that deliver users' transport needs through a single interface of a service provider. It combines different transport modes to offer a tailored mobility package, like a monthly mobile phone contract. This interpretation encompasses some of the core characteristics of MaaS: customer's need-based, service bundling, cooperativity and interconnectivity in transport modes and service providers. Cox (2015) adds to this definition, by emphasizing the similarity with the telecommunication sector. Being based on the same definition, Finger, Bert and Kupfer (2015) envisioned MaaS to integrate transport modes through the internet.

Holmberg, Collado, Sarasini and Williander (2016) emphasized the role of subscription in MaaS, giving the user the possibility to plan his/her journey, in terms of booking and paying the several transport modes that might be required, all in one service. To access the service, travelers will be asked to register or make an account. At a first level, this is to make booking and payment easier, as the concept envisions a 'seamless' com- bination of all transportation modes and a 'Mobility Aggregator' that gathers and sells all services through a single smartphone app, allowing easy fare payment and one-stop billing (CIVITAS, 2016). Based on the traveller's needs, he/she can have the choice of 'pay-as-you-go' or pre/post pay, considering his/her registration and a monthly subscription. At a second stage, subscription results in personalisation, framing mobility services around traveller's preferences, which is one important advantage that is absent from conventional public transport services and thus not covering passenger's needs which might result in inconvenience (Atasoy, Ikeda, Song, \& Ben-Akiva, 2015). More specifically, tailoring the bundles to the heterogeneous needs of the subscribers (i.e. preferences in mode choice) is beneficial for both users and transport providers usually referred to as collaborative customisation or personalisation (Hietanen, 2014; Kamargianni, Matyas, Li, \& Schäfer, 2015).

In addition to the definitions above, which emphasize the bundling and subscription aspects of MaaS, there are various other interpretations of the term that underscore other aspects. Atkins (2015) defines MaaS as a new way to provide transport, which facilitates the users to get from $A$ to $B$ by combining available mobility options and presenting them in a completely integrated manner. Thus, it is possible to consider MaaS as mobility service that is flexible, personalized and on-demand. Evidently, MaaS essential characteristic is the user-centric vision which frames the mobility service provision, a view which many authors strongly emphasize.

The key function of the internet and, more in general, of the technologies, has also been underlined in several definitions. Nemtanu, Schlingensiepen, Buretea and lordache (2016) consider the Information and Communication Technologies (ICTs) as the main component of MaaS systems. They mention the collection, transmission, process, and presentation of the information necessary for identifying the best transport solution for user's needs. ICTs also play a vital part in information integration and convergence between users, providers, and services. The emergent notion in the Internet of Things (IOT), which further accentuate the connectivity between physical objects and virtual data, is a vision of Smart transportation systems to support the Smart City vision (Sherly \& Somasundareswari, 2015). In the context of MaaS, similar emphasis stressing the importance of integrations between transport data, data infrastructure and physical transport infrastructure can also be observed (Hietanen, 2014). In Melis, Prandini, Sartori and Callegati (2016)'s interpretation, loT acts as an enabler for the integration of private and public transport. Similarly, Giesecke, Surakka and Hakonen (2016) also considered an intelligent use of ICTs as the basis for transporting persons through the combination of different means.

By providing seamless travels with accessible and affordable solutions, MaaS has a perspective to contribute toward the strategic goals to achieve integrated multimodal systems, substituting private vehicles with alterna- 
tive models (Chowdhury \& Ceder, 2016; CIVITAS, 2016; Luk \& Olszewski, 2003). Gould, Wehrmeyer and Leach (2015) envision MaaS as an opportunity to decarbonise transport sector by reducing the use of private cars and encouraging the diffusion of electric vehicles (EVs) within the city. Integrating transportation in a service like MaaS can shift the interest from private car usage to alternative modes counteracting the negative effect of current transport systems on urban contexts and the environment. However, Holmberg et al. (2016) point out the importance in setting MaaS tariff to ensure users' positive preference toward more sustainable modes, thus contributing to the sustainability vision.

Interestingly, Giesecke et al. (2016) conceptualize MaaS as a socio-technical phenomenon with sustainability as a critical aspect, thus shedding the light on the sociological level and the sustainability dimensions of the concept. This highlights the importance of users' acceptance and adoption to MaaS, as well as its roles to transform their habits and behaviours to meet their travel needs in a sustainable way. Accordingly, other authors consider sustainability and user perspective as the core elements of MaaS concept.

In the interpretation of König, Eckhardt, Aapaoja, Sochor and Karlsson (2016), MaaS offers need-based and customized mobility solutions for the users with the goal of achieving a more sustainable transport. This change of focus considers the social context to fulfil users' needs and environmental aspect while addressing the challenge of urban mobility. Implementing and delivering innovative services like MaaS will help to enhance accessibility and equity through a shift from ownership-based to access-based transportation. More specifically, a wide range of alternative modes and customized mobility services is expected to have societal value, increasing accessibility in reaching places and in the ability to utilize transport modes. Exploring the current use of shared-mobility, it is believed that these options can be the solutions for residents of low-density areas, as well as an affordable solution for low-income households (CIVITAS, 2016).

Other definitions considered the user-centred perspective from an operational point of view (Ghanbari, Álvarez San-Jaime, Casey, \& Markendahl, 2015; Kamargianni et al., 2016; Rantasila, 2016). The main goal of MaaS systems is to provide seamless door-to-door mobility for users. This is made feasible by the technological advances, the cooperation of different operators, the bundling of several transport modes. Things have to be done in a smarter and more efficient way and by the full deployment of ICT and a stronger cooperation between public and private transport providers, Maas can result in a better allocation of resources and services, with the citizen as an end-user (Hietanen, 2014).

\subsection{Proposed Core Characteristics of Maas}

Based on the literature review on definitions of MaaS in the previous section and further research about rel- evant innovative mobility services and ideas, we summarized the core characteristics that should be apparent when implementing such concept in practice. Table 1 presents those elements, without implying any hierarchical ranking.

\section{An Overview of Existing MaaS Schemes}

In this section, we present an overview of 12 selected MaaS schemes from around the world. Table 2 presents an analysis of these pilots and case studies that have been implemented in the context of MaaS and are described in accordance with the core characteristics defined in the previous section. Selecting these MaaS schemes was the outcome of a thorough review of literature and research. The list could be more extended than the one presented; we are aware of schemes such as Kätevä Seinäjoki (Seinäjoki, Finland), Mobility broker (Aachen, Germany), Mobility Mixx (Netherlands), Open Mobility (Berlin, Germany), Radiuz Total Mobility (Netherlands), Reisbalans (Netherlands), Stadtwerke PlusCard (Münster, Germany), Stuttgart-Services (Stuttgart, Germany), SwissPass Plus (Switzerland), Switchh (Hamburg, Germany), Tripkey (Netherlands), Ylläs Around (Ylläs area, Finland), and other schemes included in MaaSiFie project (König et al., 2016) and Kamargianni et al. (2016). However, the scope of this paper highlights the importance of the main characteristics that should complement MaaS concepts and applications. Therefore, the selection process made necessary to exclude those case studies or pilots that lack the majority of this set of attributes, thus we present the schemes that were once conceptualized, designed and implemented covering at a certain degree most of these aspects.

The majority (eleven) of these schemes are from Europe and one from the United States. Eight of these are operational schemes, three are pilot schemes, and one is a scheme that was planned but cancelled before its operation even began (SHIFT). Three of these schemes have already ceased their operations. While there are large variations in the criteria, certain patterns can be observed. For example, public transport is nearly always included as part of the transport modes offered (eleven). Additionally, bike sharing (seven current, one planned), car sharing (eight current, one planned), and taxi (ten current, one planned) are included in most of these schemes. Certain schemes also include rental car (three), parking (six), and regional transport (six), as well as peerto-peer car rental (one), and permit to congestion charging zone (one).

Other apparent trends: pay-per-use is mostly offered as a tariff option (seven), all schemes offer their platform through smart phone apps, while two schemes also provide web alternatives. Next, most services provide real-time information, trip planning, booking, ticketing, and payment functionalities. Some also include perks, such as push information for service alerts, integrated invoice, access to municipality services, freight services 
Table 1. Description of MaaS' core characteristics based on literature review.

\section{Core Characteristic Description}

A goal of MaaS schemes is to encourage the use of public transport services, by bringing together

1. Integration of transport modes multi-modal transportation and allowing the users to choose and facilitating them in their intermodal trips. Following transport modes may be included: public transport, taxi, car-sharing, ride-sharing, bike-sharing, car-rental, on-demand bus services. Envisioning a service beyond the urban boundaries, it will embrace also long-distance buses and trains, flights, and ferries.

MaaS platform offers users two types of tariffs in accessing its mobility services: "mobility 2. Tariff option package" and "pay-as-you-go". The package offers bundles of various transport modes and includes a certain amount of $\mathrm{km} /$ minutes/points that can be utilized in exchange for a monthly payment. The pay-as-you-go charges users according to the effective use of the service.

MaaS relies on a digital platform (mobile app or web page) through which the end-users can access to all the necessary services for their trips: trip planning, booking, ticketing, payment, and

3. One platform real-time information. Users might also access to other useful services, such as weather forecasting, synchronization with personal activity calendar, travel history report, invoicing, and feedback.

MaaS ecosystem is built on interactions between different groups of actors through a digital platform: demanders of mobility (e.g. private customer or business customer), a supplier of transport services (e.g. public or private) and platform owners (e.g. third party, PT provider,

4. Multiple actors authority). Other actors can also cooperate to enable the functioning of the service and improve its efficiency: local authorities, payment clearing, telecommunication and data management companies.

Different technologies are combined to enable MaaS: devices, such as mobile computers and

5. Use of technologies smartphones; a reliable mobile internet network (WiFi, 3G, 4G, LTE); GPS; e-ticketing and e-payment system; database management system and integrated infrastructure of technologies (i.e. IoT).

6. Demand

orientation

MaaS is a user-centric paradigm. It seeks to offer a transport solution that is best from customer's perspective to be made via multimodal trip planning feature and inclusion of demand-responsive services, such as taxi.

7. Registration requirement The end-user is required to join the platform to access available services. An account can be valid for a single individual or, in certain cases, an entire household. The subscription not only facilitates the use of the services but also enables the service personalisation.

Personalisation ensures end users' requirements and expectations are met more effectively and efficiently by considering the uniqueness of each customer. The system provides the end-user

8. Personalisation with specific recommendations and tailor-made solutions on the basis of her/his profile, expressed preferences, and past behaviors (e.g. travel history). Additionally, they may connect their social network profiles with their MaaS account.

Customisation enables end users to modify the offered service option in according to their preferences. This can increase MaaS' attractiveness among travelers and its customers'

9. Customisation satisfaction and loyalty. They may freely compose a specified chained trip or build their mobility package with a different volume of usage of certain transport modes to better achieve their preferred travel experiences.

(planned), and real-time congestion monitoring. Additionally, a third party is the service aggregator for the majority of these schemes (seven), three other schemes have PT-providers as their aggregators and two rely on their local authorities. All of them utilised GPS technology, nine cases employ ePay, two offer smart card for payments and one eWallet, which can also be used to pay for other services.

While several apps allow unregistered users to use basic functionalities like route planning, all of them required users' registration to access the service, make a booking and customise the service to their needs. More variations can be observed across the personalisation and customisation criterion. Still, certain common features, such as storing of past or favourited trips and selection of preferred modes can be discerned.

In addition to the core characteristics identified in Table 2, we established three MaaS attributes through the review of case studies, they are:

1. Decision influence-Certain MaaS schemes have features to influence users' trip decisions, ranges from a less active approach, such as SMILE's comparison of $\mathrm{CO}_{2}$ emission by each mode to a more active approach in UbiGo, which promotes PT mode, and an incentive-based of Whim, which re- 
Table 2. Summary of MaaS schemes reviewed in this study.

\begin{tabular}{|c|c|c|c|c|c|c|}
\hline $\begin{array}{l}\text { Scheme } \\
\text { (Area) }\end{array}$ & $\begin{array}{l}\text { TransitApp } \\
\text { (USA, UK, } \\
\text { Canada, Europe, } \\
\text { Australia) }\end{array}$ & $\begin{array}{l}\text { Optymod } \\
\text { (Lyon, France) }\end{array}$ & $\begin{array}{l}\text { Mobility } \mathbf{2 . 0} \\
\text { services } \\
\text { (Palma, Spain) }\end{array}$ & $\begin{array}{l}\text { SHIFT-Project } \\
100 \\
\text { (Las Vegas, USA) }\end{array}$ & $\begin{array}{l}\text { UbiGo } \\
\text { (Gothenburg, } \\
\text { Sweden) }\end{array}$ & $\begin{array}{l}\text { Mobility Shop } \\
\text { (Hannover, } \\
\text { Germany) }\end{array}$ \\
\hline $\begin{array}{l}\text { Status } \\
\text { (Year) }\end{array}$ & $\begin{array}{l}\text { Operational } \\
(2012-)\end{array}$ & $\begin{array}{l}\text { Operational } \\
(2012-)\end{array}$ & $\begin{array}{l}\text { Pilot } \\
(2013-)\end{array}$ & $\begin{array}{l}\text { Planned } \\
(2013-2015)\end{array}$ & $\begin{array}{l}\text { Pilot } \\
(2013-2014)\end{array}$ & $\begin{array}{l}\text { Operational } \\
(2014-)\end{array}$ \\
\hline $\begin{array}{l}\text { Transport } \\
\text { modes and } \\
\text { related } \\
\text { services }\end{array}$ & $\begin{array}{l}\text { PT (Inc. local } \\
\text { ferry) } \\
\text { Bike sharing } \\
\text { Car sharing } \\
\text { Taxi } \\
\text { Ride-hailing }\end{array}$ & $\begin{array}{l}\text { PT } \\
\text { Bike sharing } \\
\text { Regional train } \\
\text { Parking }\end{array}$ & $\begin{array}{l}\text { PT } \\
\text { Bike sharing } \\
\text { Taxi }\end{array}$ & $\begin{array}{l}\text { Bike sharing } \\
\text { Car sharing } \\
\text { Taxi } \\
\text { Shared shuttle }\end{array}$ & $\begin{array}{l}\text { PT } \\
\text { Bike sharing } \\
\text { Car sharing } \\
\text { Car rental } \\
\text { Taxi }\end{array}$ & $\begin{array}{l}\text { PT } \\
\text { Car sharing } \\
\text { Taxi } \\
\text { Regional trains }\end{array}$ \\
\hline $\begin{array}{l}\text { Tariff } \\
\text { option }\end{array}$ & Pay-per-use & None & Pay-per-use & $\begin{array}{l}\text { Monthly } \\
\text { tariff }\end{array}$ & $\begin{array}{l}\text { Monthly } \\
\text { tariff }\end{array}$ & $\begin{array}{l}\text { Fixed monthly } \\
\text { membership } \\
\text { to access } \\
\text { discounted tariff }\end{array}$ \\
\hline Platform & App/Web & App & App/Web & App & App & App \\
\hline $\begin{array}{l}\text { Available } \\
\text { functionalities }\end{array}$ & $\begin{array}{l}\text { Real time info. } \\
\text { Trip planning } \\
\text { Booking (shared } \\
\text { modes/Taxi) } \\
\text { Payment (bike } \\
\text { sharing) } \\
\text { Service alerts } \\
\text { Departure alarms } \\
\text { Stop notifications }\end{array}$ & $\begin{array}{l}\text { Real time info. } \\
\text { congestion } \\
\text { Prediction } \\
\text { Trip planning } \\
\text { Booking (bike } \\
\text { sharing) } \\
\text { Service alerts } \\
\text { Plane's arrival- } \\
\text { departure time } \\
\text { info }\end{array}$ & $\begin{array}{l}\text { Real time info. } \\
\text { Trip planning } \\
\text { Service alerts } \\
\text { Real time } \\
\text { congestion } \\
\text { monitor }\end{array}$ & $\begin{array}{l}\text { Trip planning } \\
\text { Booking } \\
\text { Payment } \\
\text { Invoicing }\end{array}$ & $\begin{array}{l}\text { Trip planning } \\
\text { Booking } \\
\text { Ticketing } \\
\text { Payment } \\
\text { Invoicing } \\
24 \text { hr customer } \\
\text { service phone } \\
\text { line }\end{array}$ & $\begin{array}{l}\text { Real time info. } \\
\text { Booking } \\
\text { Ticketing } \\
\text { Payment } \\
\text { Invoicing } \\
\text { Service alerts }\end{array}$ \\
\hline $\begin{array}{l}\text { Type of actors } \\
\text { involved } \\
\text { Service } \\
\text { aggregator }\end{array}$ & $\begin{array}{l}\text { Public and } \\
\text { private actors } \\
\text { 3rd party }\end{array}$ & $\begin{array}{l}\text { Public actors } \\
\text { Local } \\
\text { authority }\end{array}$ & $\begin{array}{l}\text { Public and } \\
\text { private actors } \\
\text { Local authority }\end{array}$ & $\begin{array}{l}\text { Private actor } \\
3 r d \text { party }\end{array}$ & $\begin{array}{l}\text { Public and } \\
\text { private actors } \\
\text { 3rd party }\end{array}$ & $\begin{array}{l}\text { Public and } \\
\text { private actors } \\
\text { PT provider }\end{array}$ \\
\hline $\begin{array}{l}\text { Use of } \\
\text { technologies }\end{array}$ & $\begin{array}{l}\text { GPS / ePay (bike } \\
\text { sharing only) }\end{array}$ & GPS & GPS & GPS / ePay & $\begin{array}{l}\text { GPS / Smart } \\
\text { card }\end{array}$ & $\begin{array}{l}\text { GPS / ePay / } \\
\text { Smart card }\end{array}$ \\
\hline $\begin{array}{l}\text { Demand } \\
\text { orientation }\end{array}$ & Yes & Yes & Yes & Yes & Yes & Yes \\
\hline $\begin{array}{l}\text { Registration } \\
\text { requirement }\end{array}$ & $\begin{array}{l}\text { Yes, for } \\
\text { booking and } \\
\text { customisation }\end{array}$ & $\begin{array}{l}\text { Yes, for } \\
\text { customisation }\end{array}$ & $\begin{array}{l}\text { Yes, for } \\
\text { booking and } \\
\text { customisation }\end{array}$ & NA & $\begin{array}{l}\text { Yes, for usage } \\
\text { and } \\
\text { customisation }\end{array}$ & $\begin{array}{l}\text { Yes, for usage } \\
\text { and } \\
\text { customisation }\end{array}$ \\
\hline Personalisation & $\begin{array}{l}\text { Store regular } \\
\text { and preferred } \\
\text { routes } \\
\text { Saved location }\end{array}$ & $\begin{array}{l}\text { Input personal } \\
\text { address, } \\
\text { preferable } \\
\text { modes, and } \\
\text { ownership of } \\
\text { bicycle }\end{array}$ & $\begin{array}{l}\text { Store } \\
\text { favourite } \\
\text { trips }\end{array}$ & $\begin{array}{l}\text { Automatically } \\
\text { Optimised } \\
\text { trip planner }\end{array}$ & NA & $\begin{array}{l}\text { Store favourites } \\
\text { trips and recall } \\
\text { previous trip }\end{array}$ \\
\hline Customisation & $\begin{array}{l}\text { Minimised } \\
\text { walking option } \\
\text { Disabled certain } \\
\text { service/modes } \\
\text { Link with } \\
\text { calendar and } \\
\text { personal contact }\end{array}$ & $\begin{array}{l}\text { Select service } \\
\text { subscription } \\
\text { for news }\end{array}$ & $\begin{array}{l}\text { Enable } \\
\text { certain map } \\
\text { sections and } \\
\text { accessibility } \\
\text { map people } \\
\text { with special } \\
\text { needs }\end{array}$ & $\begin{array}{l}\text { Mobility budget } \\
\text { with Top-up }\end{array}$ & $\begin{array}{l}\text { Mobility } \\
\text { budget with } \\
\text { Top-up and } \\
\text { roll over }\end{array}$ & $\begin{array}{l}\text { Possibility to } \\
\text { create individual } \\
\text { mix of } \\
\text { transportation } \\
\text { Booking and } \\
\text { payment } \\
\text { cancelation }\end{array}$ \\
\hline
\end{tabular}


Table 2. Summary of MaaS schemes reviewed in this study (continued).

\begin{tabular}{|c|c|c|c|c|c|c|}
\hline $\begin{array}{l}\text { Platform } \\
\text { (Area) }\end{array}$ & $\begin{array}{l}\text { Smile } \\
\text { (Vienna, Austria) }\end{array}$ & $\begin{array}{l}\text { Tuup } \\
\text { (Turku Region, } \\
\text { Finland) }\end{array}$ & $\begin{array}{l}\text { My Cicero } \\
\text { (Italy) }\end{array}$ & $\begin{array}{l}\text { Moovel } \\
\text { (Germany) }\end{array}$ & $\begin{array}{l}\text { Whim } \\
\text { (Helsinki, } \\
\text { Finland) }\end{array}$ & $\begin{array}{l}\text { WienMobil Lab } \\
\text { (Vienna, } \\
\text { Austria) }\end{array}$ \\
\hline $\begin{array}{l}\text { Status } \\
\text { (Year) }\end{array}$ & $\begin{array}{l}\text { Pilot } \\
(2014-2015)\end{array}$ & $\begin{array}{l}\text { Operational } \\
(2015-)\end{array}$ & $\begin{array}{l}\text { Operational } \\
(2015-)\end{array}$ & $\begin{array}{l}\text { Operational } \\
(2016-)\end{array}$ & $\begin{array}{l}\text { Operational } \\
(2016-)\end{array}$ & $\begin{array}{l}\text { Based on Smile } \\
\text { project } \\
(2015-2016)\end{array}$ \\
\hline $\begin{array}{l}\text { Transport } \\
\text { modes and } \\
\text { related } \\
\text { services }\end{array}$ & $\begin{array}{l}\text { PT } \\
(\mathrm{e}-) \text { Bike sharing } \\
\text { (e-)Car sharing } \\
\text { Taxi } \\
\text { Parking garages } \\
\text { Charging stations } \\
\text { Regional trains } \\
\text { and ferry }\end{array}$ & $\begin{array}{l}\text { PT } \\
\text { Bike sharing } \\
\text { Car sharing } \\
\text { Car rental } \\
\text { P-2-P car rent } \\
\text { Taxi and shared } \\
\text { taxi } \\
\text { Parking rent } \\
\text { Freight service* }\end{array}$ & $\begin{array}{l}\text { PT } \\
\text { Taxi* } \\
\text { Parking spaces } \\
\text { Permit for } \\
\text { urban } \\
\text { congestion } \\
\text { charging zone } \\
\text { Regional rail } \\
\text { and bus }\end{array}$ & $\begin{array}{l}\text { PT } \\
\text { Bike sharing } \\
\text { Car sharing } \\
\text { Taxi } \\
\text { Ferry } \\
\text { Regional rail }\end{array}$ & $\begin{array}{l}\text { PT } \\
\text { Rental car } \\
\text { Taxi } \\
\text { Regional rail } \\
\text { Bike sharing* } \\
\text { Car sharing* }\end{array}$ & $\begin{array}{l}\text { PT } \\
\text { Bike-sharing } \\
\text { Car-sharing } \\
\text { Taxi } \\
\text { Parking garages }\end{array}$ \\
\hline $\begin{array}{l}\text { Tariff } \\
\text { option }\end{array}$ & Pay-per-use & Pay-per-use & Pay-per-use & Pay-per-use & $\begin{array}{l}\text { Three monthly } \\
\text { packages and } \\
\text { pay-per-use }\end{array}$ & Pay-per-use \\
\hline Platform & App & App & App & App & App & App \\
\hline $\begin{array}{l}\text { Available } \\
\text { functionalities }\end{array}$ & $\begin{array}{l}\text { Real time info. } \\
\text { Trip planning } \\
\text { Booking (shared } \\
\text { modes / Taxi / } \\
\text { Regional train) } \\
\text { Ticketing } \\
\text { Payment } \\
\text { Invoicing } \\
\text { Service alerts }\end{array}$ & $\begin{array}{l}\text { Real time info. } \\
\text { Trip planning } \\
\text { Booking } \\
\text { Ticketing } \\
\text { Payment (for PT, } \\
\text { taxi, and shared } \\
\text { taxi) }\end{array}$ & $\begin{array}{l}\text { Real time info. } \\
\text { Trip planning } \\
\text { Booking } \\
\text { Ticketing } \\
\text { Payment } \\
\text { Invoicing } \\
\text { Municipality } \\
\text { services }\end{array}$ & $\begin{array}{l}\text { Real time info } \\
\text { Trip planning } \\
\text { Booking } \\
\text { Ticketing } \\
\text { Payment } \\
\text { Invoicing }\end{array}$ & $\begin{array}{l}\text { Real time info. } \\
\text { Trip planning } \\
\text { Booking } \\
\text { Ticketing } \\
\text { Payment } \\
\text { Invoicing }\end{array}$ & $\begin{array}{l}\text { Real time info. } \\
\text { Trip planning } \\
\text { Booking } \\
\text { Payment } \\
\text { Invoicing }\end{array}$ \\
\hline $\begin{array}{l}\text { Type of actors } \\
\text { involved } \\
\text { Service } \\
\text { aggregator }\end{array}$ & $\begin{array}{l}\text { Public and } \\
\text { private actors } \\
\text { PT provider }\end{array}$ & $\begin{array}{l}\text { Public and } \\
\text { private actors } \\
\text { Third party }\end{array}$ & $\begin{array}{l}\text { Public and } \\
\text { private actors } \\
\text { Third party }\end{array}$ & $\begin{array}{l}\text { Public and } \\
\text { private actors } \\
\text { Third party }\end{array}$ & $\begin{array}{l}\text { Public and } \\
\text { private actors } \\
\text { Third party }\end{array}$ & $\begin{array}{l}\text { Public and } \\
\text { private actors } \\
\text { PT provider }\end{array}$ \\
\hline $\begin{array}{l}\text { Use of } \\
\text { technologies }\end{array}$ & GPS / ePay & $\begin{array}{l}\text { GPS / ePay } \\
\text { (PayiQ) }\end{array}$ & $\begin{array}{l}\text { GPS / ePay / } \\
\text { e-Wallet }\end{array}$ & GPS / ePay & GPS / ePay & GPS / ePay \\
\hline $\begin{array}{l}\text { Demand } \\
\text { orientation }\end{array}$ & Yes & Yes & Yes & Yes & Yes & Yes \\
\hline $\begin{array}{l}\text { Registration } \\
\text { requirement }\end{array}$ & $\begin{array}{l}\text { Yes, for usage } \\
\text { and } \\
\text { customisation }\end{array}$ & $\begin{array}{l}\text { Yes, for usage } \\
\text { and } \\
\text { customisation }\end{array}$ & $\begin{array}{l}\text { Yes, for usage } \\
\text { and } \\
\text { customisation }\end{array}$ & $\begin{array}{l}\text { Yes, for usage } \\
\text { and } \\
\text { customisation }\end{array}$ & $\begin{array}{l}\text { Yes, for usage } \\
\text { and } \\
\text { customisation }\end{array}$ & $\begin{array}{l}\text { Yes, for } \\
\text { booking and } \\
\text { customisation }\end{array}$ \\
\hline Personalisation & $\begin{array}{l}\text { Optimised trip } \\
\text { plan to user's } \\
\text { profile (i.e. } \\
\text { annual ticket, } \\
\text { subscription and } \\
\text { membership) }\end{array}$ & $\begin{array}{l}\text { Optimised } \\
\text { travel plan } \\
\text { based on user's } \\
\text { daily agenda }\end{array}$ & $\begin{array}{l}\text { Store types } \\
\text { of ticket } \\
\text { Record and } \\
\text { share journey }\end{array}$ & $\begin{array}{l}\text { Store favourites } \\
\text { routes } \\
\text { Personalised } \\
\text { notification on } \\
\text { disruptions }\end{array}$ & $\begin{array}{l}\text { Calendar } \\
\text { synchronization } \\
\text { Personal info } \\
\text { sharing } \\
\text { Social } \\
\text { interaction }\end{array}$ & $\begin{array}{l}\text { Save personal } \\
\text { mobility profile } \\
\text { Store car \& bike } \\
\text { sharing } \\
\text { membership }\end{array}$ \\
\hline Customisation & $\begin{array}{l}\text { Enable mode } \\
\text { filtering based } \\
\text { on cost, time, } \\
\text { and } \mathrm{CO}_{2} \text { footprint }\end{array}$ & $\begin{array}{l}\text { Preferred } \\
\text { modes, based } \\
\text { on cost and } \\
\mathrm{CO}_{2} \text { footprint }\end{array}$ & $\begin{array}{l}\text { Preferred } \\
\text { modes and } \\
\text { payment } \\
\text { Top-up }\end{array}$ & $\begin{array}{l}\text { Link with social } \\
\text { media accounts } \\
\text { Booking } \\
\text { cancellation }\end{array}$ & $\begin{array}{l}\text { Cancelation } \\
\text { Change of } \\
\text { subscription } \\
\text { Top-up }\end{array}$ & $\begin{array}{l}\text { Preferred } \\
\text { modes, based } \\
\text { on cost, time, } \\
\text { and } \mathrm{CO}_{2} \\
\text { footprint }\end{array}$ \\
\hline
\end{tabular}

Note: *Planned service. 
wards users for their 'green' trips. These features can be beneficial in ensuring MaaS positive contribution to sustainability. On the other hand, it also points toward a need for a monitoring system to ensure that such feature is utilized for societal benefits.

2. The inclusion of other services-SMILE included access to parking, park and ride service, e-vehicle, and regional ship demonstrates the result of including a broad range of stakeholders in MaaS. Tuup's inclusion of Piggybaggy, a crowdsourcing freight transport service and My Cicero's municipality services are also unique examples how MaaS can open the possibility for other transport related services.

3. Mobility 'currency'-Whim is the only scheme considered here that employs this feature, which can be a step toward a truly integrated multimodal transport system. It enables users to customise their monthly mobility budget to best suit their preferences and not 'locked in' by any sunk cost, such as annual PT subscription or car rental membership. On the other hand, it also increases platform provider influence toward pricing of service. A Whim point purchase through its most expensive subscription (389€ for 10,000 points) is more than $50 \%$ cheaper than a Whim point purchase through its most basic package ( $89 €$ for 1,000 points). The economy of scale of such basic commodity can have implications on equity aspects.

\section{State of the Art and Future Challenges with MaaS}

In this section, we examine the perspective implications of MaaS based on our proposed core characteristics in three areas of transport practice: travel demand modeling, supply-side analysis, and designing business model.

\subsection{Demand-Side Modelling}

Transport modelling is important and essential for estimating travel demand and offering valuable information to policy makers and transport planners. During the years, several modelling approaches have been explored and formulated. In travel demand modelling, conventional models were aggregate in nature and the dominant approach was the four-step modelling process. Dissatisfaction with trip-based models, policy needs for detailed sociodemographic information for the trip at individual/household level but mostly the behavioural inadequacy of this approach has led to the emergence of disaggregate forecasting models (Bhat \& Koppelman, 1999). Both supply and demand models have evolved from static to dynamic capturing travel behaviour in terms of time-dependent conditions and information, and from an aggregate to a disaggregate representation of travel, focusing on the heterogeneity of individual traveling (Ben-Akiva, Bottom, Gao, Koutsopoulos, \& Wen, 2007).
Moving to activity-based approach, new aspects are of crucial importance: integrity, interdependencies between trips of the same trip chain or household, higher temporal and spatial aggregation and a strong behavioural basis, as engaging in an activity in fact 'represents' a dynamic interaction of household needs, tasks, and constraints (Rasouli \& Timmermans, 2014a). One of the first types of activity-based models were the constraints models, examining the feasibility of agendas with a great emphasis on the role of spatial-temporal constraints on daily travel behaviour. Then, the second approach in activity-based modelling was the econometric one, based on discrete choice models and on the principle of utility maximisation to model pattern formation. Following those, rule-based models (also known as computational process models) have developed, creating activity schedules based on heuristics and decision rules. However, more extended approaches have emerged including time-space prisms and constraints and agent-based modelling (Bhat \& Koppelman, 1999; Timmermans, Arentze, \& Joh, 2002).

All these activity-based model systems have been built upon the assumption that travel decisions are made under conditions of certainty. This hypothesis can be considered not realistic, as the state of transportation systems is affected by a variety of uncertainty factors and decision makers don't have a perfect knowledge about their choice set. So, in order to improve the accuracy and reliability of such models, interesting theories and models of human choice and decision-making under risk and uncertainty have been explored and developed in travel behaviour analysis (Rasouli \& Timmermans, 2014b). Among these, prospect theoretic models (Kahnema \& Tversky, 1979) and regret-based models (Chorus, Arentze, \& Timmermans, 2008) are gaining an interest in travel behaviour research in recent years, serving as a valuable alternative to the dominant utilitymaximisation models. The introduction of uncertainty in the decision-making process should be accompanied by the identification and exploration of other drivers of travellers' choice behaviours, which must be considered in modelling travel demand for innovative mobility services like MaaS in the dynamic and complex context of the smart city.

MaaS is a user-centered service adopting the advances of technology and ICT to offer various mobility solutions to customers, conceptualising travel differently. In this new context, people will have a wide list of options to choose from, based on public and private transport modes, multiple needs and preferences, and a service which allows them to pursue more activities within the same timeline (multitasking). Activity-based modelling techniques are considered crucial for understanding how individual and households organise their daily activities. At the same time, ICT and smartphones have contributed positively to models used to predict the sequence between activity and travel episodes. However, attempting to deliver innovative services like MaaS, requires exten- 
sions in current activity-based modelling, considering the more dynamic context of modern lifestyle, social influence, ICT, responses to travel recommendation systems, attitudes and subjective considerations and the increasing degree of uncertainty. Thus, a critical reflection on how to expand current activity-based models and their underlying theories and choice models is needed to better capture the comprehensive nature of the travel behaviour and decision-making process related to MaaS. Other theories and models originally formulated in different fields, like marketing, behavioural economics, social psychology and technology and innovation, might be explored for their appropriateness in modelling the demand side of Maas.

To the best of our knowledge, as Maas is an emerging trend and its implementation in the real world is still limited, there is a very small number of studies examining the travel demand modelling for MaaS. Sochor, Strömberg and Karlsson $(2014,2015)$ studied the changes in travel behaviours, users' mode choices and level of satisfactions by collecting and analysing data on a six-month field operational test of UbiGo. Data was collected from participating and non-participating households, throughout a mixed-methods approach, to identify the interests and the obstacles for joining UbiGo. Results reveal the main motives of adopting and using MaaS (i.e. curiosity, convenience, flexibility) and how they change over time. Findings also indicate that the participants could be considered as innovators or early adopters, confirming the need to integrate innovation theory in travel demand modelling applied to MaaS. Afterward, Sochor, Strömberg and Karlsson (2016) focus on the application of Roger's diffusion of innovation theory as a useful tool for travel behaviour change in a sustainable direction.

In their study about the development of a mobility assistance system, Hilgert, Kagerbauer, Schuster and Becker (2016) develop a new activity-generation module in the agent-based, microscopic travel demand model mobiTopp (Mallig, Kagerbauer, \& Vorisch, 2013) to gain insights about changes in travel behaviour and daily mobility patterns due to the use of customized mobility services. This model used a utility function base on user's favourite criteria (e.g. time, cost, and, preferred modes of transport). Binomial and multinomial logit models were estimated to analyse activity and travel related decisions at different steps, using the data of the German Mobility Panel (MOP) (Hilgert et al., 2016). The implementation of this travel demand model is still ongoing, but it is expected to quantify the effects of mobility assistance on individual daily travel behaviour and travel demands.

As underlined by the European Commission (2016), existing quantitative studies on MaaS impacts on users travel behaviour are not yet developed. For a better understanding of this emergent phenomenon and its implications, studying and modelling user's acceptance factors and travel-related choices represent an urgent area for further research.

\subsection{Supply-Side Modelling}

The supply side focuses on the modes of transport offered and covers both design and operations. The impact that MaaS has on the supply side can be perceived in the early name that this concept was given in the EU policy scene in 2009: the "Fifth Mode" (Schade, Krail, \& Kühn, 2014). There are different reasons why MaaS represents such a disruptive concept for the supply side despite aiming "merely" at a complete integration of the existing modes of transport, and we aim at mentioning the most relevant ones.

The integration that MaaS represents has been triggered by the number and variety of new on-demand transportation services that have appeared in the transportation arena. Among these services, we encounter shared services, namely car-sharing and bike-sharing. The one-way configuration of these shared services (the car or bike can be left at the destination and not necessarily at the initial pick-up point), is the one that allows for more flexibility and, therefore, the most suitable for MaaS. The major challenges in designing such services are vehicle fleet optimization and relocation strategies (Cepolina, Farina, \& Pratelli, 2014). A step forward needed to bring them further under a MaaS ecosystem would be to take the integrated MaaS supply network into account in these relocation strategies. The effect of autonomous vehicles is another aspect that needs to be considered and that will ease relocation efforts. Research suggests that autonomous cars can rebalance themselves in the network and coordinate their actions at a system-wide level (Zhang, Spieser, Frazzoli, \& Pavone, 2011), solving some of the possible system level problems of car-sharing and Litman (2017) suggests that automatic car-sharing/taxi schemes will become a reality in 2030-40s suggesting a positive impact of automated vehicles on MaaS.

Other on-demand transportation services that will play an important role in MaaS are demand responsive on-demand services. They can be classified into individual services, such as regular taxis or Uber-like services, and collective services (e.g. Kutsuplus and Uberpool). The major challenge in modelling this kind of services lies in designing the routing strategy for the vehicles. The routing algorithm to deal with this routing problem has been widely studied and is known in optimisation as the dial-a-ride problem (DARP), which is a generalisation of the Travelling Salesman Problem (TSP). Heuristic search algorithms have been proven to provide optimal solutions for these problems. To control and plan the requests with the desired level of service in the pick-up and drop-off times, the supplier uses the time window approach, as studied, for example, in (Mahmoudi \& Zhou, 2016). As an extension of the DARP, the Integrated Dial-aride Problem (IDARP) includes the integration of demand responsive services with fixed route services. This mode integration scheme shows how the combination of different modes of transport can improve the transportation 
network by exploiting the benefits of each service, as it is aimed at in MaaS ecosystems. The optimisation objective of such integration focuses on maximising passenger utility (Wilson, Weissberg, \& Hauser, 1976), maximising the service capacity (Liaw, White, \& Bander, 1996), or any other operational or service related aspect.

Further than the mentioned IDARP, which focuses on the integration of demand responsive services and fixed route service only, the intermodal mobility planning needs to consider all available services. Currently, it is mainly approached applying concepts from the realm of graph theory and constraint-satisfaction-problems (Masuch, Marco, \& Keiser, 2013), but available research on this topic is still limited.

Even if the integration of transport modes is the first requirement in MaaS schemes, payment integration and ICT integration are also main components in the supply integration that MaaS offers, as it has also been reported by Kamargianni et al. (2016). Regarding payment integration, smart cards have been its main enabler. Smart cards offer a large amount of information that can be used both in the planning and operation stages. Nevertheless, better algorithms are still needed to validate the data, and new modelling methods will be needed, such as the Totally Disaggregate Approach, to deal with the high detailed level of resolution provided by these information sources (Pelletier, Trépanier, \& Morency, 2011). MaaS enablers will need to consider these aspects since the integration of all available modes will intensify these problems. The longitudinal data provided by these integrated payment methods can be used to better understand travel patterns and better localise the critical points for the supply-demand optimisation. Privacy of data is another principal source of concern about the smart card (and alike) payment methods (Dempsey, 2008). More recently, mobile payments (considered as virtual smart cards) are also being studied (Di Pietro, Guglielmetti Mugion, Mattia, Renzi, \& Toni, 2015).

Integration of modes and the payment for their usage would not be useful in MaaS if the whole information of these services were not readily available. This is why the advancements in ICT platforms are a major aspect in the development of MaaS systems. The integrated and realtime multimodal information provided to the traveler can update a traveller's perception of the travel alternatives dynamically (Chorus, Molin, \& Van Wee, 2006) and challenge the perceptions of their perceived utility related to non-car modes (Kenyon \& Lyons, 2003), inducing modal shift. This information is acquired from unifying very different types and sources of the data, and the data aggregation is still one of the main technical challenges when dealing with intermodal algorithms (Masuch et al., 2013). The integration of information is of vital importance and a pillar in Maas: it is what the end user receives and upon which the whole supply network builds upon. Other than that, good governance and business models need to be assured to ensure the correct functioning of such a complex system.

\subsection{Governance and Business Model to Match Supply and Demand}

An implementation of MaaS can have significant impacts to the existing business model of public transport, especially on the level of integration. An increase in the required level of integration can pose a dilemma for public transport providers in their decisions related to integration with other operators. Traditionally, public transport services are usually provided by monopoly or multiservice providers benefiting from economies of scope and scale (Viton, 1992; Farsi, Fetz, \& Filippini, 2007). Apart from the conventional provision of services and its pros and cons, public transport providers might benefit from MaaS, which seems an advanced version of integrated public transport services. Technically, integration of services may be realised by using so-called platform technology, which facilitates interactions between travellers and suppliers of transport services in an improved or smarter way (Ballon, 2009; Gawer, 2014). Economists perceive platforms as markets which mediate transactions across different customer groups or 'sides'. Multisided platform (MSP) is a model for MaaS. Besides few practical examples to date, experience can be gained from other industries such as ICT, telecommunications, and airlines industry (Hagiu \& Wright, 2015).

A crucial characteristic of MSP is the presence of the network externalities (also known as network effects and demand-side economies). Direct and indirect externalities are two types of network effects (Shapiro \& Varian, 1999). The direct network effect is that utility of a product increases by growing number of users on the same side of the platform, usually for product interconnecting people such as communication technologies (i.e. telephone, e-mail, games). The indirect network effect is defined as an effect in which an increase in the number of users on one side is beneficial to other sides of the platform. The indirect effects that arise between users and developers of games stem from two sources: 1) a membership effect, which members on one side enjoy greater benefits of having more members on the other side to potentially transact with, independent of the nature of the product and 2) a usage effect, which users have greater benefits from using better complementary products (Rochet \& Tirole, 2003).

Additionally, platforms create value by coordinating these services through providing information about the prices and qualities of the services. For example, Uber offers a platform that matches travellers demanding a trip and car owners that want to supply this trip (Gawer, 2014; Hagiu, 2014). MSPs reduce search and transaction costs. Search costs are costs incurred by the multiple sides before they interact, to determine the best "trading partners." (Hagiu \& Wright, 2015). Nevertheless, there are certain challenges in establishing a platform, such as the chicken and egg problem (getting both sides to use the platform) and gaining a critical mass of users on both sides in the right proportions to guarantee acceptable 
added-value and sustainable growth of platforms (Hagiu, 2014; Jullien \& Caillaud, 2003).

Few studies investigated the application of MSP in public transport. Finger et al. (2015) explain the concept of MaaS with practical examples. Their evaluation covers the challenges in operationalising mobility platforms, including getting a critical mass and regulatory challenge to provide a market opportunity for platforms. Sochor et al. (2015) discuss the matches and mismatches of customers' expectations with delivered services during experimenting UbiGo. Although the project's expectations were met on increased of transport options, easier payment, tracking expenditures, and reduced need for private car ownership, there are also several mismatches. Firstly, the revenue generated by the service was below expectation. This is due to the below-than-expected car rental and car sharing services usage by its users. Additionally, regulation on the reselling of PT service prevented UbiGo from purchase PT services and make a profit of them. Secondly, the users needed to pay a minimum amount in advance and they perceived it less flexible. A study by Meurs \& Timmermans (2017) define and discussed the features and challenges for successful implementation of MSP. They explain the concept of network externalities as an important feature of MSP. Increasing the number of car shares make the platform more attractive for other users (direct network effect) and If more transport providers join the platform, the utility of travellers will increase due to more options being offered (indirect network effect). Additionally, the chicken-and-egg problem and achieving the critical mass are mentioned as challenges in implementing MSP in PT. König et al. (2016) define business ecosystem of MaaS and develop a framework to value chain of different MaaS schemes. A similar work is done by Kamargianni \& Matyas (2017), classifying different actors based on the relationships with MaaS providers. They conclude that the distribution model of MaaS differs from the prevalence way of mobility services' provision which is one of the distinctive features of MSP. The MaaS providers aggregate the offering services by mobility providers. Thus, MaaS is not only about the integration of mobility services, but also requires a complete restructuring of the supply chain of mobility service providers.

\section{Conclusion}

The novelty and fuzzy natures of MaaS make it a challenge to ascertain what MaaS is, its implications and how to address them. We distilled a set of core characteristics of Maas from a literature review and used this to present an overview of selected MaaS schemes that fulfil more or less these elements. The review reveals that while there is a diversity in attributes, such as personalisation, customisation, tariff options and platform aggregators, certain patterns can be observed among the schemes considered, such as the modes included in the services, available basic functionality (real time informa- tion, trip planning, booking, and ticketing) and employed technologies (GPS, E-ticket, and E-payment). It also appears that certain schemes go further to offer perk features, such as trip disruption warning and synchronization with personal agenda.

The assessment also reveals certain attributes unique to case studies, such as features that can influence trip decision, the inclusion of other services, such as freight transport and municipality services, and the use of a mobility currency. These characteristics may add to enhance the proposed framework but requires additional case studies to confirm if they are essential parts or perks of MaaS.

We then look at the possible implications of these core characteristics toward demand modelling, supply modelling, and governance in transport practices. We established the state of art in the mentioned fields and proposed the probable enhancements needed to deliver such an innovative service like MaaS. Our suggestions include extensions in current activity-based modelling, improvements in optimisation of vehicle fleet and routing for DRT, and enhancements on integrations, among others. We also point out challenges in the implementation of the multi-sided platforms, such as chicken and egg problem and achieving a critical mass of user.

The findings in this study provide a point of reference for MaaS definition, a description framework of relevant schemes, and a direction toward further works in the three areas. It should also be relevant to other researches or activities related to Mobility-as-a-Service. Additional enhancements to this analysis can be made by adding more schemes into the description framework, which can further reveal differences, similarities, and unique characteristics of the services.

\section{Acknowledgments}

The authors would like to appreciate Prof. Henk Meurs and Prof. dr. Ir. Vincent Marchau for the kind support, comments and suggestions. We also thank our anonymous reviewers for their valuable contributions.

\section{Conflict of Interests}

The authors declare no conflict of interests.

\section{References}

Atasoy, B., Ikeda, T., Song, X., \& Ben-Akiva, M. E. (2015). The concept and impact analysis of a flexible mobility on demand system. Transportation Research Part C: Emerging Technologies, 56, 373-392. doi:10.1016/j.trc.2015.04.009

Atkins. (2015). Journeys of the future. Introducing Mobility as a Service. Retrieved from http://www. atkinsglobal.com/ /media/Files/A/Atkins-Corporate /uk-and-europe/uk-thought-leadership/reports/Jour neys of the future_300315.pdf 
Ballon, P. (2009). Control and value in mobile communications: A political economy of the reconfiguration of business models in the European mobile industry (January 14, 2009). doi:10.2139/ssrn.1331439

Ben-Akiva, M., Bottom, J., Gao, S., Koutsopoulos, H. N., \& Wen, Y. (2007). Towards disaggregate dynamic travel forecasting models. Tsinghua Science and Technology, 12(2), 115-130. doi:10.1016/S10070214(07)70019-6

Bhat, C. R., \& Koppelman, F. S. (1999). Activity-based modeling of travel demand. In R. Hall (Ed.), Handbook of transportation science (pp. 35-61). New York: Springer. doi:10.1007/978-1-4615-5203-1_3

Cepolina, E. M., Farina, A., \& Pratelli, A. (2014). Carsharing relocation strategies: A state of the art. In A. Pratelli (Ed.), Public mobility systems. doi:10.2495/ 978-1-84564-908-1/010

Chorus, C. G., Arentze, T. A., \& Timmermans, H. J. P. (2008). A random regret-minimization model of travel choice. Transportation Research Part B: Methodological, 42(1), 1-18. doi:10.1016/j.trb.2007. 05.004

Chorus, C. G., Molin, E. J., \& Van Wee, B. (2006). Use and effects of advanced traveller information services (ATIS): A review of the literature. Transport Reviews, 26(2), 127-149. doi:10.1080/ 01441640500333677

Chowdhury, S., \& Ceder, A. (2016). Users' willingness to ride an integrated public-transport service: A literature review. Transport Policy, 48, 183-195. doi:10.1016/j.tranpol.2016.03.007

CIVITAS. (2016). Mobility-as-a-Service: A new transport model. Retrieved from http://civitas.eu/content/civ itas-insight-18-mobility-service-new-transport-model

Cox, N. C. J. (2015). Estimating demand for new modes of transportation using a context-aware stated preference survey (Doctoral Dissertation). Massachusetts Institute of Technology, USA.

Dempsey, P. (2008). Privacy issues with the use of smart cards. Legal Research Digest (pp. 2-25). Washington DC: Transportation Research Board. doi:10.17226/23104

Di Pietro, L., Guglielmetti Mugion, R., Mattia, G., Renzi, M. F., \& Toni, M. (2015). The integrated model on mobile payment acceptance (IMMPA): An empirical application to public transport. Transportation Research Part C: Emerging Technologies, 56, 463-479. doi:10.1016/j.trc.2015.05.001

European Commission. (2016). HORIZON 2020-Work Programme 2016-2017. Smart, green and integrated transport (Vol. 2017). Retrieved from https://ec. europa.eu/research/participants/data/ref/h2020/wp /2016_2017/main/h2020-wp1617-transport_en.pdf

Farsi, M., Fetz, A., \& Filippini, M. (2007). Economies of scale and scope in local public transportation. Journal of Transport Economics and Policy, 41(3), 345-361.

Finger, M., Bert, N., \& Kupfer, D. (2015). 3rd European Intermodal Transport Regulation Summary "Mobility-
as-a-Service: from the Helsinki experiment to a European model?" (Technical report, European Transport Regulation Observer No. 2015/01). Retrieved from http://fsr.eui.eu/Documents/WorkshopPaper/Trans port/2015/150309MaaSObserver.pdf

Gawer, A. (2014). Bridging differing perspectives on technological platforms: Toward an integrative framework. Research Policy, 43(7), 1239-1249. doi:10.1016/j.respol.2014.03.006

Ghanbari, A., Álvarez San-Jaime, O., Casey, T., \& Markendahl, J. (2015). Repositioning in value chain for smart city ecosystems: A viable strategy for historical telecom actors. In 2015 Regional Conference of the International Telecommunications Society (ITS). Los Angeles, USA.

Giesecke, R., Surakka, T., \& Hakonen, M. (2016). Conceptualising Mobility as a Service. A user centric view on key issues of mobility services. In Eleventh International Conference on Ecological Vehicles and Renewable Energies (EVER). Monte Carlo, Monaco.

Gould, E., Wehrmeyer, W., \& Leach, M. (2015). Transition pathways of e-mobility services. WIT Transactions on Ecology and The Environment, 194, 349-359. doi:org/10.2495/SC150311

Hagiu, A. (2014). Strategic decisions for multisided platforms. MIT Sloan Management Review, 55(2), 71-80. Retrieved from http://sloanreview.mit.edu/ article/strategic-decisions-for-multisided-platforms/

Hagiu, A., \& Wright, J. (2015). Marketplace or reseller? Management Science, 61(1), 184-203. doi:10.1287/ mnsc. 2014.2042

Hietanen, S. (2014). "Mobility as a Service"-The new transport model? Eurotransport, 12(2), 2-4.

Hilgert, T., Kagerbauer, M., Schuster, T., \& Becker, C. (2016). Optimization of individual travel behavior through customized mobility services and their effects on travel demand and transportation systems. Transportation Research Procedia, 19, 58-69. doi:10.1016/j.trpro.2016.12.068

Holmberg, P.-E., Collado, M., Sarasini, S., \& Williander, M. (2016). Mobility as a Service-MaaS. Describing the framework (Final report MaaS framework). Göteborg: Viktoria Swedish ICT.

Jullien, B., \& Caillaud, B. (2003). Chicken \& egg: Competition among intermediation service providers. Journal of Economics, 34(2), 309-328.

Kahnema, D., \& Tversky, A. (1979). Prospect theory: An analysis of decision under risk. Econometrica, 47(2), 263-292. doi:10.2307/1914185

Kamargianni, M., Li, W., Matyas, M., \& Schäfer, A. (2016). A critical review of new mobility services for urban transport. Transportation Research Procedia, 14, 3294-3303. doi:10.1016/j.trpro.2016.05.277

Kamargianni, M., \& Matyas, M. (2017). The business ecosystem of Mobility as a Service. Paper presented at the 96th Transportation Research Board Annual Meeting, Washington DC, USA.

Kamargianni, M., Matyas, M., Li, W., \& Schäfer, A. 
(2015). Feasibility study for "Mobility as a Service" concept in London. London: UCL Energy Institute, Department for Transportation. Retrieved from https://www.bartlett.ucl.ac.uk/energy/docs/fsmaas-compress-final

Kenyon, S., \& Lyons, G. (2003). The value of integrated multimodal traveller information and its potential contribution to modal change. Transportation Research Part F: Traffic Psychology and Behaviour, 6(1), 1-21. doi:10.1016/S1369-8478(02)00035-9

König, D., Eckhardt, J., Aapaoja, A., Sochor, J. \& Karlsson, M. (2016). Business and operator models for Mobility as a Service (MaaS) (Deliverable 3 to the MAASiFiE project). Brussels: Belgium.

Liaw, C. F., White, C. C., \& Bander, J. (1996). A decision support system for the bimodal dial-a-ride problem. IEEE Transactions on Systems, Man, and Cybernetics Part A:Systems and Humans, 26(5), 552-565. doi:10.1109/3468.531903

Litman, T. (2017). Autonomous vehicle implementation predictions Implications for transport planning. Victoria, BC: Victoria Transport Policy Institute.

Luk, J., \& Olszewski, P. (2003). Integrated public transport in Singapore and Hong Kong. Road and Transport Research, 12(4), 41-51.

Mahmoudi, M., \& Zhou, X. (2016). Finding optimal solutions for vehicle routing problem with pickup and delivery services with time windows: A dynamic programming approach based on state-space-time network representations. Transportation Research Part B: Methodological, 89, 19-42. doi:10.1016/ j.trb.2016.03.009

Mallig, N., Kagerbauer, M., \& Vorisch, P. (2013). mobiTopp-A modular agent-based travel demand modelling framework. Procedia Computer Science, 19, 854-859. doi:10.1016/j.procs.2013.06.114

Masuch, N., Marco, L., \& Keiser, J. (2013). An open extensible platform for intermodal mobility assistance. 19(Ant), 396-403. doi:10.1016/j.procs.2013.06.054

Melis, A., Prandini, M., Sartori, L., \& Callegati, F. (2016). Public transportation, loT, trust and urban habits. In F. Bagnoli et al. (Eds.), International Conference on Internet Science (pp. 318-325). Cham: Springer.

Meurs, H., \& Timmermans, H. (2017). Mobility as a Service as a multi-sided market: Challenges for modeling . Paper presented at the 96th Transportation Research Board Annual Meeting, Washington DC, USA.

Nemtanu, F., Schlingensiepen, J., Buretea, D., \& Iordache, V. (2016). Mobility as a Service in smart cities. In A. Zbuchea \& D. Nikolaidis (Eds.), Responsible entrepreneurship-Vision, development and ethics: Proceedings of the 9th International conference for entrepreneurship, innovation and regional development. June 23-24, 2016 Bucharest, Romania (pp. 425-435). Bucharest, Romania: Comunicare.ro.

Pelletier, M.-P., Trépanier, M., \& Morency, C. (2011). Smart card data use in public transit: A literature review. Transportation Research Part C: Emerg- ing Technologies, 19(4), 557-568. doi:10.1016/ j.trc.2010.12.003

Rantasila, K. (2016). The impact of Mobility as a Service concept to land use in Finnish context. In 2015 International Conference on Sustainable Mobility Applications, Renewables and Technology, SMART 2015. doi:10.1109/SMART.2015.7399229

Rasouli, S., \& Timmermans, H. (2014a). Activity-based models of travel demand: Promises, progress and prospects. International Journal of Urban Sciences, 18(1), 31-60. doi:10.1080/12265934.2013.835118

Rasouli, S., \& Timmermans, H. (2014b). Applications of theories and models of choice and decision-making under conditions of uncertainty in travel behavior research. Travel Behaviour and Society, 1(3), 79-90. doi:10.1016/j.tbs.2013.12.001

Rochet, J.-C., \& Tirole, J. (2003). Platform competition in two-sided markets. Journal of the European Economic Association, 1(4), 990-1029. doi:10.1111/j.1467-8462.2013.12020.x

Schade, W., Krail, M., \& Kühn, A. (2014). New mobility concepts: Myth or emerging reality? In Transport Research Arena-TRA 2014, 5th Conference-Transport Solutions: From Research to Deployment.

Shapiro, C., \& Varian, H. R. (1999). Information rules: A strategic guide to the network economy. Cambridge, MA: Harvard Business Press.

Sherly, J., \& Somasundareswari, D. (2015). Internet of things based smart transportation systems. International Research Journal of Engineering and Technology, 2(7), 1207-1210.

Sochor, J., Strömberg, H., \& Karlsson, I. C. M. (2014). Travelers' motives for adapting a new, innovative travel service: Insights from the UbiGo Field Operational Test in Gothenburg, Sweden. In 21s World Congress on Intelligent Transportation Systems, Detroit, September 7-11, 2014 (pp. 1-13). Detroit, MI: USA.

Sochor, J., Strömberg, H., \& Karlsson, I. C. M. (2015). Implementing Mobility as a Service challenges in integrating user, commercial, and societal perspectives. Transportation Research Record, 4(2536), 1-9. doi:10.3141/2536-01

Sochor, J., Strömberg, H., \& Karlsson, I. C. M. (2016). Trying out mobility as a service experiences from a field trial and implications for understanding demand. Paper presented at the 95th Transportation Research Board Annual Meeting, Washington DC, USA.

Timmermans, H., Arentze, T., \& Joh, C. H. (2002). Analysing space-time behaviour: New approaches to old problems. Progress in Human Geography, 26(2), 175-190.

Viton, P. A. (1992). Consolidations of scale and scope in urban transit. Regional Science and Urban Economics, 22(1), 25-49.

Wilson, N., Weissberg, R. W., \& Hauser, J. (1976). Advanced dial-a-ride algorithms research project (No. R76-20 Final Report). Cambridge, MA: Mas- 
sachusetts Institute of Technology.

Zhang, R., Spieser, K., Frazzoli, E., \& Pavone, M. (2011).

Models, algorithms, and evaluation for autonomous mobility-on-demand systems. In American Control Conference (ACC) 2015, Chicago (pp. 2573-2587). IEEE.

\section{About the Authors}

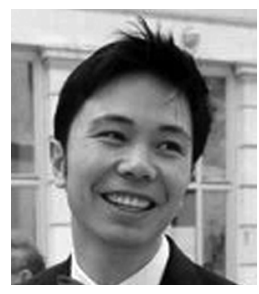

Peraphan Jittrapirom is a post-doc researcher in the Smart Cities' Responsive Intelligent Public Transport System (SCRIPTS) project at Radboud University. His research interests are transport policy and planning, system dynamics, foresight, and planning practice under deep uncertainty. He has a wide range of experience working with the private, public, and international sectors. He is also a keen advocate of the living lab. He founded the Chiang Mai's Urban Mobility Lab, an urban planning co-creation platform in 2015.

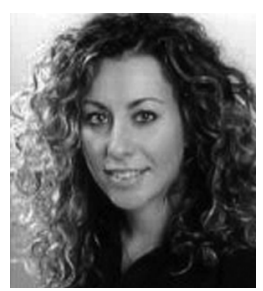

Valeria Caiati is a PhD candidate at Eindhoven University of Technology. Her PhD project aims at exploring and modelling the demand for innovative demand-driven mobility services based on platform technology (e.g. Mobility as a Service). Before starting her PhD studies, she worked in Italy for five years in international consultancy firms and research institutes, being involved in projects related to smart cities and smart mobility. She has a background in environmental engineering, with a specialization on climate change and sustainability issues.

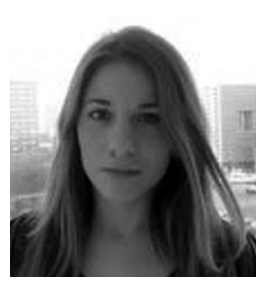

Anna-Maria Feneri is a PhD student at the Eindhoven University of Technology. She holds a Diploma in Urban Planning \& Spatial Development from the School of Engineering, Aristotle University (Greece) and a MSc in Environmental \& Infrastructure Planning from the Faculty of Spatial Sciences, University of Groningen (The Netherlands). During her academic research so far she has focused on spatial analysis, smart city development and urban quality of life. She applied her academic knowledge in the private sector before joining the Urban Planning Group at TU/e.

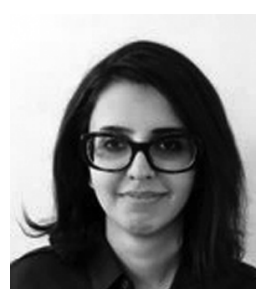

Shima Ebrahimi is a PhD candidate at the Radboud University. Her PhD project title is Governance of Mobility Services. Before starting her PhD, she completed a MSc in Engineering and Policy Analysis (EPA) at TU Delft. She also obtained a BSc in agricultural engineering, and a MSc degree in economic system programming from the University of Tehran and Shahid Beheshti University in Iran.

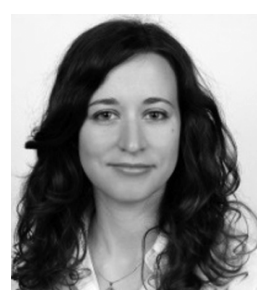

María J. Alonso González is a PhD candidate at TU Delft. Her PhD project involves the forecast and evaluation of New Mobility Services in a Hybrid Public Transport System. Her aim is to improve the offered public transport service and network performance. She has a Master's level Double Degree in Civil Engineering from the Technical University of Madrid (UPM) and the Technical University of Munich (TUM), with special focus on transportation.

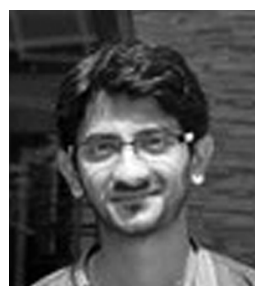

Jishnu Narayan is a PhD candidate in the Department of Transport and Planning at TU Delft. His research involves optimal network design for hybrid public transport systems combining traditional line/schedule based public transport systems with innovative mobility systems and investigating its implications. He obtained his Master's degree from IIT Kanpur (India) in Transportation Engineering and Bachelor's from NIT Calicut (India) in Civil Engineering. He worked for two years in transportation/infrastructure firms in India prior to his $\mathrm{PhD}$. 